\title{
Extragenital donovanosis: three cases from Western Australia
}

\author{
J SCHNEIDER,* J O’SHEA,* L R FINLAY-JONES, $\dagger \mathrm{K}$ M MITCHELL, $\dagger$ AND A N ROBERTS $\dagger$
}

From the *Sir Charles Gairdner Hospital, Perth, and the †State Health Laboratory Services, Queen Elizabeth II Medical Centre, Nedlands, Western Australia.

SUMMARY Three unusual cases of extragenital donovanosis are described. The presenting features were ulceration of the anterior abdominal wall, discharging sinuses of the neck, and an abscess of bone, respectively. Two cases were diagnosed recently and were the only instances of extragenital infection in a series of 47 patients with confirmed donovanosis diagnosed at a public health laboratory in Perth, Western Australia, between 1979 and the beginning of 1985. The third case, which occurred in 1977, was found in the records of a major teaching hospital.

\section{Introduction}

Though donovanosis is most commonly an ulcerogranulomatous condition affecting the genital region, the disease may have an important visceral component that may give rise to difficulties in diagnosis and management. These difficulties may be increased in non-endemic areas where doctors and pathologists may overlook the possibility of unusual forms of this disease. The rare case described by Spagnolo et al, ${ }^{1}$ in which primary donovanosis affected both axillae and upper arms, showed some of these problems.

References to extragenital donovanosis have come from various parts of the world including India, ${ }^{2-5}$ the United States of America, ${ }^{6}$ Papua New Guinea, ${ }^{78}$ and Africa. ${ }^{9}$ Brigden and Guard alluded to three major extragenital manifestations in donovanosis: (1) blood borne dissemination to distant organs such as bones, joints, liver, spleen, and lungs; (2) contiguous spread from the genital region into adjacent pelvic organs; and (3) probable spread by autoinnoculation to remote skin sites as well as to the mucosal surfaces of the lips, gums, and pharynx. ${ }^{10}$

The three cases described each show one of these occurrences and highlight the diagnostic difficulties that may ensue if extragenital donovanosis is not considered in the differential diagnosis of unusual lesions. The presenting feature in one patient was of

Address for reprints: Dr J Schneider, Sir Charles Gairdner Hospital, Queen Elizabeth II Medical Centre, Verdun Street, Nedlands, Western Australia 6009, Australia

Accepted for publication 18 November 1985 recurrent abscesses in the abdominal wall necessitating numerous admissions to hospital. The second patient was febrile and unwell and presented with deep sinuses close to the angles of both jaws. The third patient had a lytic lesion of the right ilium, which was at first incorrectly diagnosed as a gumma.

\section{Case reports}

CASE 1

A part aboriginal man aged 25 first presented with a small abscess in the left groin in September 1982 at a Western Australian country hospital $800 \mathrm{~km}$ north of Perth. Initial treatment was by incision and drainage. No organisms were cultured and there was little response to gentamicin, clindamycin, and benzylpenicillin. One month later, he presented again with a second abscess in the left iliac fossa. In November 1982 he was transferred to a teaching hospital in Perth where, in addition to a scar in the left suprapubic region, a fluctuant swelling in the left iliac fossa was noted. The swelling was incised and a large amount of watery fluid drained out. Again no organisms were cultured. A track leading deep into the pelvis was found and explored. A sinogram showed a contrast filled cavity deep to the left iliac fossa lesion. An intravenous pyelogram showed no abnormality. Despite continuing drainage of fluid from his abscess, the patient refused further treatment in hospital.

In May 1983 the patient was again seen and was found to have an abscess, measuring $15 \mathrm{~cm}$ by $5 \mathrm{~cm}$, situated $5 \mathrm{~cm}$ deep in the left iliac fossa and a small wound $2 \mathrm{~cm}$ in diameter in the left suprapubic region. 
Rectal examination showed another abscess $6 \mathrm{~cm}$ to the left of the anus. In addition he then had two penile lesions. Slough was removed from the abdominal lesions (fig 1) and the rectal abscess was drained internally. Biopsy specimens from both abscess cavities and both penile lesions were sent for histopathology and culture. After these procedures the patient stated that occasionally he passed air through his penis, and on one occasion during micturition urine emerged from the lower of his two ulcers. These symptoms resolved spontaneously.

He was transferred to the Sir Charles Gairdner Hospital and, apart from having had primary syphilis in 1974, which had been fully treated, his medical history was not remarkable. He was single, worked as a labourer, smoked and drank heavily, and gave no history of homosexual contact. One penile lesion on the prepuce was $1 \mathrm{~cm}$ in diameter, moderately tender, and surrounded by a raised rolled margin of tissue (fig $2)$. The other adjacent lesion was smaller and ulcerated, resembling a syphilitic chancre. He had no associated lymphadenopathy. The clinical features of

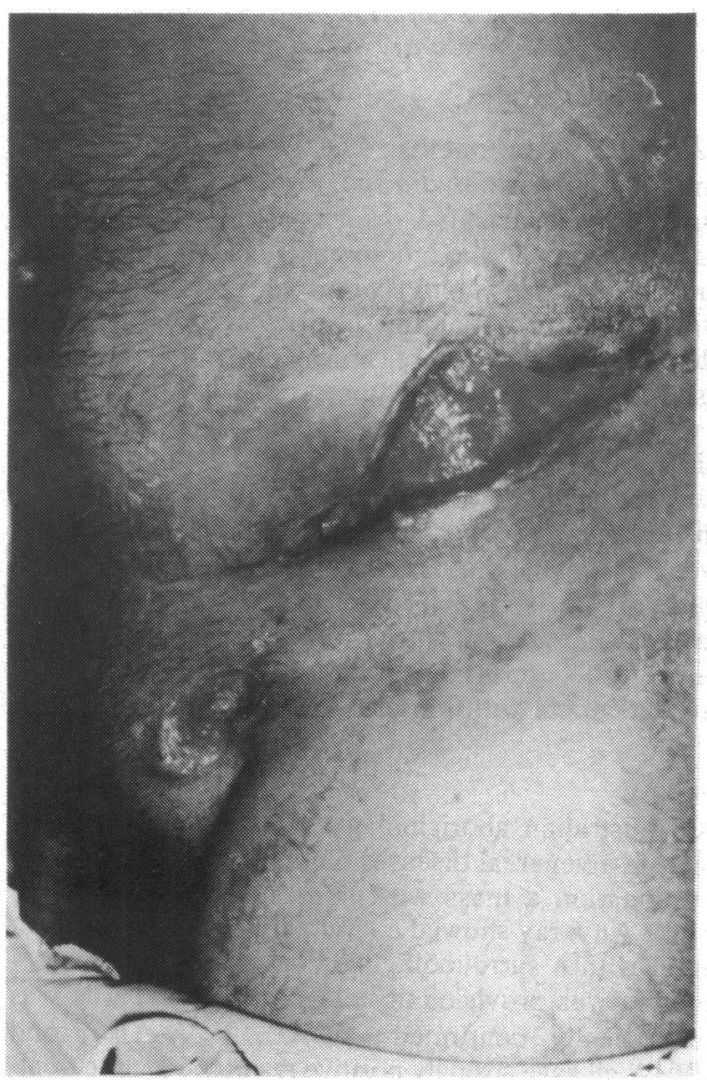

FIG 1 Abdominal wall and inguinal lesions of case 1 after removal of slough. the large penile lesion were in keeping with donovanosis, and a confident clinical diagnosis was made before the biopsy results became available. Laboratory investigations were non-contributory apart from an erythrocyte sedimentation rate of $20 \mathrm{~mm}$ in the first hour, Giardia lamblia cysts in the stool, slightly increased gamma glutamyl transferase activity of 69 IU/1 (normal 10-50 IU/1), and syphilis serology test results consistent with previous infection (Venereal Disease Research Laboratory (VDRL) test negative, Treponema pallidum haemagglutination assay (TPHA) 4+, fluorescent treponemal antibody absorbed (FTA-ABS) test IgG 4+, and FTA-ABS test IgM negative). Histopathological examination of all biopsy specimens showed similar features. Granulation tissue contained a heavy mixed infiltrate of plasma cells, lymphocytes, and neutrophilic leucocytes accompanied by some histiocytes. Parts of the epidermis manifested pseudoepitheliomatous hyperplasia. Special stains (Giemsa, Warthin-Starry) showed organisms with the morphology of Calymmatobacterium granulomatis in the cytoplasm of the histiocytes (fig 3). An intravenous pyelogram then showed an abnormal mass in the left pelvis, which produced a lobulated impression on the pelvic portion of the left ureter, displaced the bladder to the right, and caused hydronephrosis (fig 4). There was no evidence of fistula formation. Cystoscopy showed a necrotic mound (about $1 \mathrm{~cm}$ in diameter) of granulation tissue in the left lateral dome of the bladder. No other lesion

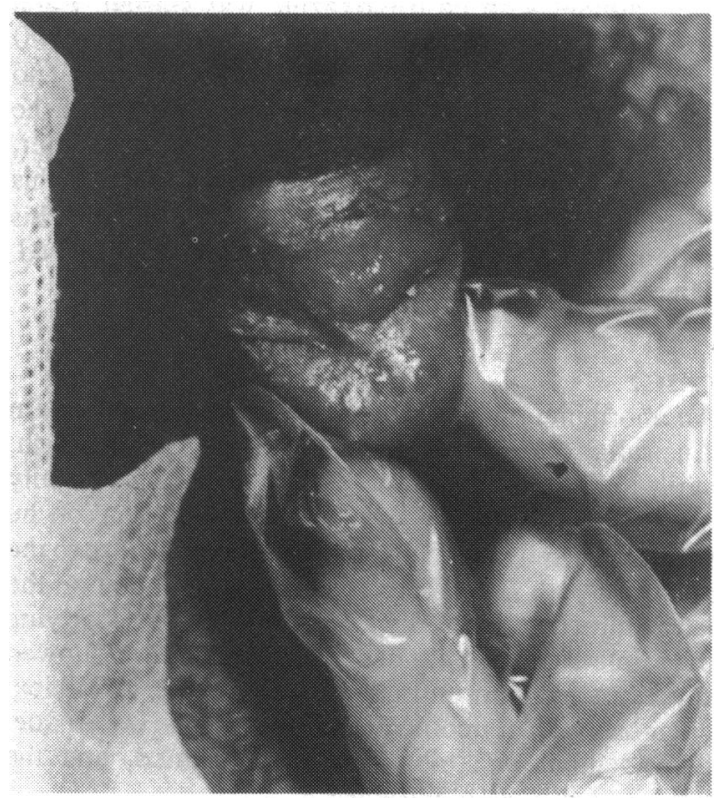

FIG 2 (Case 1.) Beefy-red moderately tender penile lesion with rolled margin. 


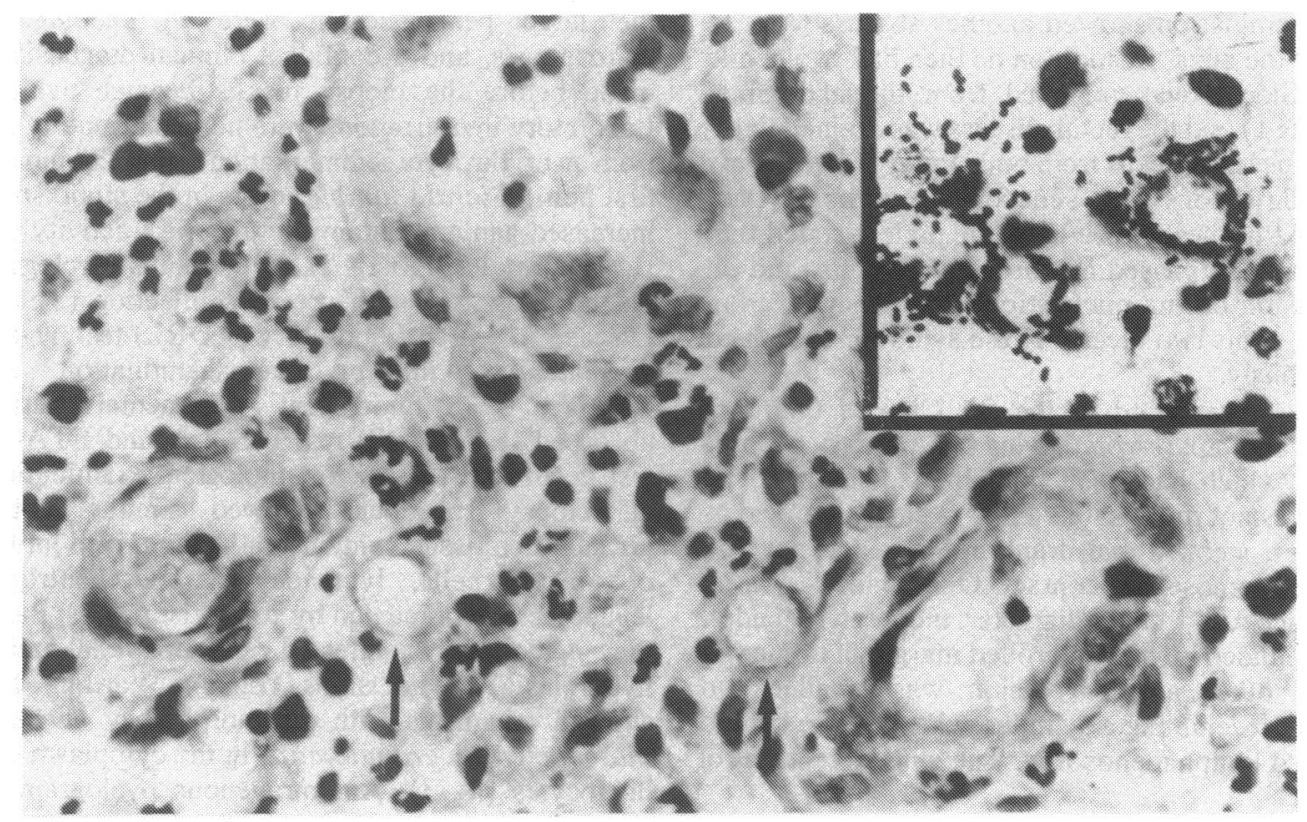

FIG 3 (Case 1.) High power photomicrograph showing neutrophils, plasma cells, and (arrowed) vacuolated histiocytes (haematoxylin and eosin). Inset: typical micro-organisms of donovanosis (Warthin-Starry, oil immersion).

could be seen. Although sigmoidoscopy disclosed no abnormality except for the incision site of his rectal abscess, a sinogram carried out on the left iliac fossa lesion showed a further track (fig 5).

Treatment with co-trimoxazole was started. Each dose (trimethoprim $160 \mathrm{mg}$, sulphamethoxazole 800 $\mathrm{mg}$ ) was given every eight hours for seven days and then every 12 hours for another 14 days before he discharged himself from hospital. Five months later, when he was readmitted to a different unit of this hospital for treatment of abdominal trauma, his abdominal ulceration and penile lesions had healed. There was no evidence of fistula formation.

\section{CASE 2}

An aboriginal man aged 28 was referred to a Western Australian regional hospital, $540 \mathrm{~km}$ east of Perth, with a six month history of multiple chronically discharging sinuses of the neck. On examination he was febrile and unwell and had two sinuses close to the angles of both jaws. These were deep, and that on the left admitted an index finger whereupon the underlying mandible could be palpated. Oral examination showed severe caries with multiple dental sinuses. A clinical diagnosis of cervicofacial actinomycosis was made. Debridement and biopsy were performed, and the wisdom teeth were extracted.

Laboratory investigations showed a severe chronic iron deficiency anaemia (haemoglobin concentration
$72 \mathrm{~g} / 1$ with neutrophilia. The VDRL test, tests for antibodies to Chlamydia spp, a neck swab, and blood cultures gave negative results. Histology showed a heavy mixed chronic inflammatory cell infiltrate with many plasma cells and scattered histiocytes. Special stains disclosed that the histiocytes contained the characteristic intracytoplasmic organisms of donovanosis.

The patient was discharged from hospital before the histological diagnosis became available, but returned two weeks later with continuing fever. Examination of the inguinal regions showed large bilateral raised weeping lesions that extended to the scrotum and the base of the penis. Further biopsy specimens were taken, and treatment with tetracycline was started. The histological appearances of these biopsy specimens were identical with those of the previous biopsy.

\section{CASE 3}

An Australian aboriginal woman aged 45 was first seen in a venereal diseases clinic, and during clinical examination a mass was palpated in the right iliac fossa. An $x$ ray showed a large lytic lesion of the right ilium with a surrounding soft tissue mass (fig 6). A gumma was provisionally diagnosed as syphilis was serologically confirmed (VDRL, FTA-ABS, and TPHA all gave strongly positive results). Routine treatment was instituted, but the patient was lost to follow up until one year later when she returned with a large, 


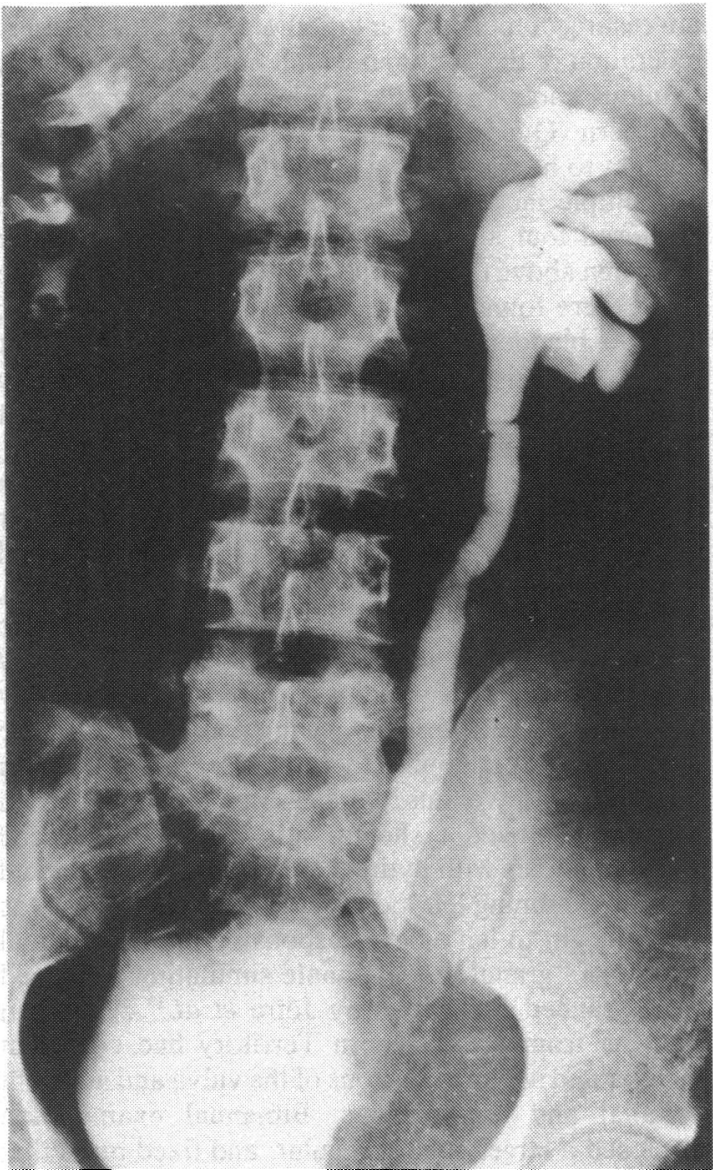

FIG 4 (Case 1.) Intravenous pyelogram showing left sided hydronephrosis and hydroureter caused by mass obstructing lower end of left ureter and displacing bladder.

hard, non-tender mass that was again palpable in the right iliac fossa. Biopsy examination to exclude malignancy was recommended. At operation at the Royal Perth Hospital a large quantity of necrotic tissue was found at the medial aspect of the right ilium, which was associated with an underlying hard bony mass. The biopsy specimens showed the typical appearances of donovanosis, and $C$ granulomatis was found. No previous evidence of genital donovanosis had been seen, but after the biopsy a chronic discharging sinus developed in the right groin. This sinus persisted with some evidence of pyogenic infection in the right wing of the ilium, despite treatment with a full course of tetracycline. The patient suggested that this "wound" was related to injury from a wooden spear (spearing is a traditional form of tribal punishment) as she described the discharge of wooden splinters. This was never substantiated. Fourteen months after biopsy the wound had healed and $x$ ray examination showed only

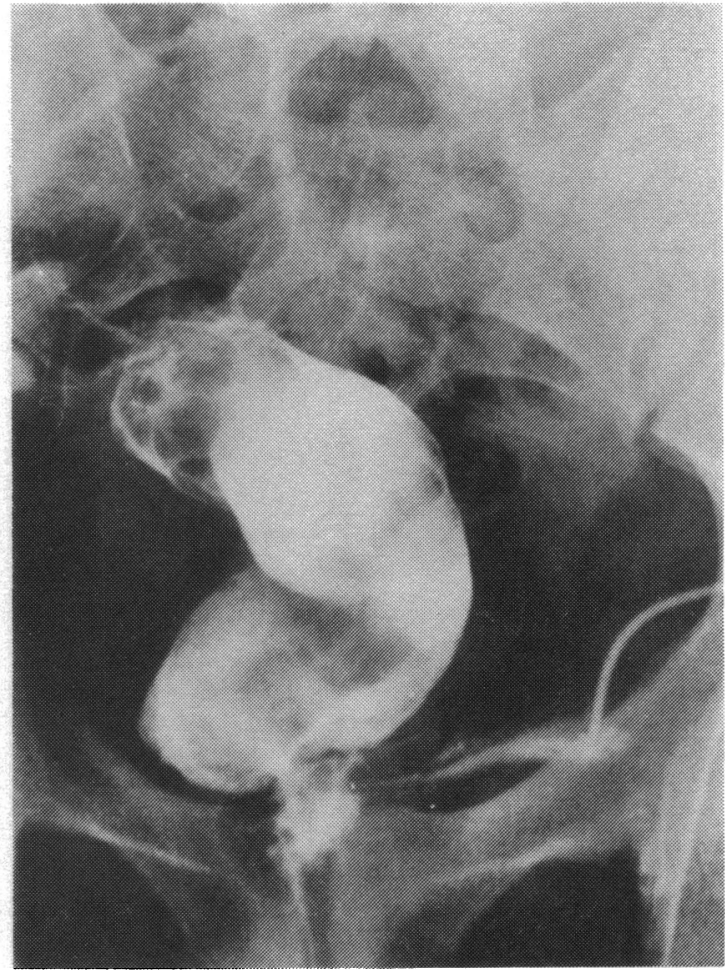

FIG 5 (Case 1.) Sinogram showing fistulous tract between lower rectum and larger of abdominal wall lesions.

minor residual changes in the right ilium.

\section{Discussion}

Our patients with extragenital manifestations warrant discussion from a number of standpoints. Only two were found in our series of 47 patients with confirmed extragenital donovanosis, the third having been found in the records of another major teaching hospital. Our series, comprising cases histologically and cytologically proved in a single laboratory during slightly more than six years, is described in another paper. ${ }^{11}$ Similar rates of extragenital infection have been reported in many other series. In the United States of America Greenblatt et al stated that extragenital lesions occured in about $6 \%$ of all patients with donovanosis; ${ }^{6}$ in India Rajam et al, quoting from experience of over 2000 patients, also observed extragential lesions in $6 \% ;^{2}$ in Papua New Guinea, Maddocks et al found no evidence of extragenital infection in 87 patients with donovanosis at the Port Moresby General Hospital but subsequently they encountered a 15 year old boy with bone lesions; ; in Northern Queensland, 


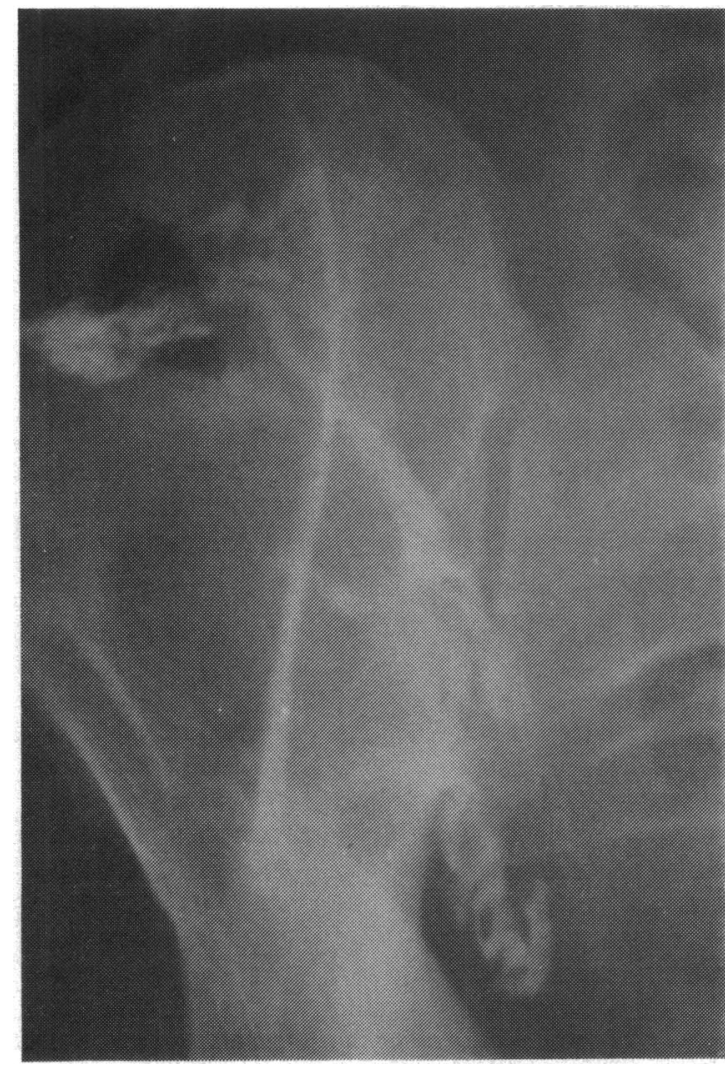

FIG 6 (Case 3.) Sinogram showing osteolytic lesion of right ilium and fistula extending into ischiorectal fossa.

Australia, Brigden and Guard stated that out of 43 cases of genital donovanosis two had extragenital infection. ${ }^{10}$ Such incidence of extragenital disease was not the experience at the Institute of Venereology in Madras, where for the past three decades 100 to 250 cases of donovanosis a year have been diagnosed but only a single case of disseminated donovanosis has been reported. ${ }^{12}$

Rajam et al asserted that destructive lesions of bones and joints were the most frequent form of metasteses, which were explicable only on the basis of haematogenous transmission. ${ }^{2}$ Osteolytic lesions present as metaphyseal deposits in long bones or multiple osteolytic cortical defects. ${ }^{13}$ In one of two patients with extragenital donovanosis reported on from Northern Queensland the features were fever, swollen knee joints, and osteolytic lesions of distal bones. ${ }^{10}$ Our case 3 had a solitary osteolytic lesion, which was probably caused by haematogenous spread, though there was the unsubstantiated possibility of trauma having caused direct spread.

Contiguous spread from the genital region into adjacent pelvic organs was probably the cause of the most interesting clinical presentation of our case 1 , namely ulceration of the abdominal wall and the formation of fistulous tracts. In another somewhat similar case from Northern Queensland this means of spread was thought to be responsible for the clinical presentation of multiple faecal fistulae. ${ }^{10}$ Abdominal examination disclosed four sinus tracks opening on to the lower abdomen above the inguinal ligament. At necropsy the tracts were found to communicate with one another, with the bladder, and with the descending colon. Our patient showed a fistula from the left lower abdomen to the lower rectum. Another feature worthy of comment in our patient was the left sided ureteric obstruction, which was probably caused by a mass of granulation tissue affecting the bladder. Donovanosis of the ureter has been described rarely. Scrimgeour et al described granulomatous infecton of the parametrium, salpinges, ovaries, and uterus that was associated with ureteric obstruction and bilateral hydronephrosis. ${ }^{14}$ Rajam et al referred to a 22 year old patient found at cystoscopy to have a large "growth" filling the trigone of the bladder and overlapping the ureteric orifices on both sides. On intravenous pyelography the patient had previously been shown to have prounced hydronephrosis with hydroureter on the right side and a non-functioning left kidney. Contiguous spread was also thought to have been responsible for the clinical picture of "granuloma inguinale simulating advanced pelvic cancer" described by Jofre et al. ${ }^{15}$ A woman aged 35 from the Northern Territory had confluent nodular and ulcerated lesions of the vulva and adjacent perianal and crural areas. Bimanual examination disclosed a large, solid, irregular, and fixed mass that filled the pelvis and extended to both lateral pelvic walls.

Cutaneous and oral lesions of donovanosis are rarely described. ${ }^{135}$ Such lesions may be either primary infections or secondary manifestations of the genital form of the disease. In the latter case, spread is probably by autoinoculation from infected clothing or fingernails. Orogenital sexual practices could also result in oral lesions, but Felman and Nikitas stated that the role of such activities "in the transmission of the oral lesions of donovanosis has not been emphasised". ${ }^{16}$ Spread by autoinoculation probably accounts for the findings in our case 2 , which was also noteworthy for the presence of sinuses discharging on the surface of the skin.

Diagnosis and treatment of donovanosis is relatively straightforward if doctors and pathologists are aware of the possibility of this disease. Typically a patient with donovanosis has superficial spreading ulceration of the genital region and no enlargement of the regional lymph nodes. We think that in the northern parts of Western Australia, where donovanosis is endemic, clincal diagnosis is common and biopsy confirmation is only sought in atypical cases or when there is a poor 
response to conventional antibiotic treatment. Diagnostic difficulties, as exemplified by our patients, can arise with extragenital lesions or if patients present in a non-endemic area. The diagnosis may be easily missed if biopsy specimens are not appropriately stained to show the characteristic micro-organisms and are therefore reported as being merely inflamed granulation tissue. It is therefore important that clinicians and pathologists are acquainted with the multiple and varied features of this disease.

\section{References}

1. Spagnolo DV, Coburn PR, Cream JJ, Azadian BS. Extragenital granuloma inguinale (donovanosis) diagnosed in the United Kingdom: a clinical, histological, and electron microscopical study. J Clin Pathol 1984;37:945-9.

2. Rajam RV, Rangiah PN, Anguli VC. Systemic donovanosis. British Journal of Venereal Diseases 1954;30:73-80.

3. Rao MS, Kameswari VR, Ramula C, Reddy CR. Oral lesions of granuloma inguinale. J Oral Surg 1976;34:1112-4.

4 Garg BR, Lal S, Bedi BMS. Donovanosis (granuloma inguinale) of the oral cavity. British Journal of Venereal Diseases 1975;51:136-7.

5. Sehgal VN, Sharma NL, Bhargava NC, Nayar M, Chandra M. Primary extragenital disseminated cutaneous donovanosis. BrJ Dermatol 1979;101:353-6.
6 Greenblatt RB, Dienst RB, Baldwin KR. Lymphogranuloma venereum and granuloma inguinale. Med Clin North $\mathrm{Am}$ 1959;43:1493-506.

7. Zigas V. Medicine from the past $-\mathbf{a}$ donovanosis project in Goilala (1951-1954). Papua New Guinea Med J 1971;14:148-9.

8. Maddocks I, Anders EM, Dennis E. Donovanosis in Papua New Guinea. British Journal of Venereal Diseases 1976; 52:190-6.

9. Bhagwandeen SB, Mottiar YA. Granuloma venereum. J Clin Pathol 1972;25:812-6.

10. Brigden $M$, Guard $\mathbf{R}$. Extragenital granuloma inguinale in North Queensland. Med J Aust 1980;2:565-7.

11. Mitchell KM, Roberts AN, Williams VM, Schneider J. Donovanosis in Western Australia. Genitourin Med 1986;62: 191-5.

12. World Health Organisation. Nongonococcal urethritis and other selected sexually transmitted diseases of public health importance. WHO Tech Rep Ser 1981;No. 660:52-6.

13. Kirkpatrick DJ. Donovanosis (granuloma inguinale): rare cause of osteolytic bone lesions. Clin Radiol 1970;21:101-5.

14. Scrimgeour EM, Sengupta SK, McGoldrick IA. Primary endometrial and endocervical granuloma inguinale (donovanosis). British Journal of Venereal Diseases 1983;59:198-201.

15. Jofre ME, Webling DD'A, James ST. Granuloma inguinale simulating advanced pelvic cancer. Med J Aust 1976;2:86973.

16. Felman YM, Nikitas JA. Sexually transmitted diseases of the oral cavity: part II. Cutis 1982;30:181-98, 254. 